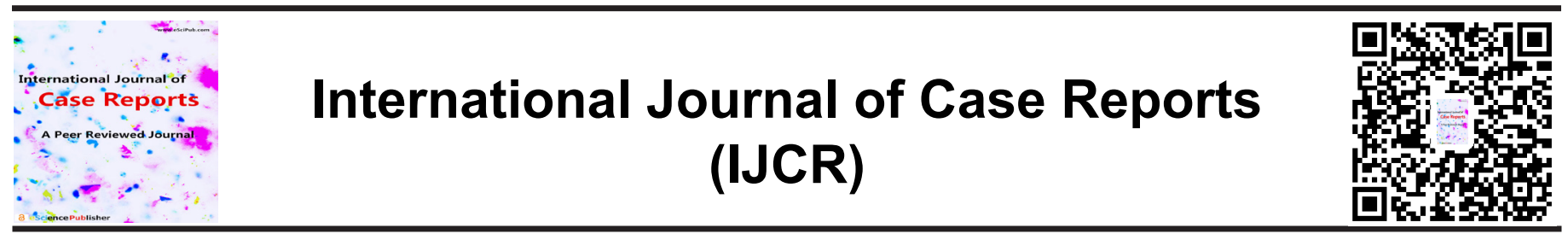

\title{
Duodenal duplication cyst: a rare cause of gastro-intestinal obstruct ion
}

\author{
S.Jarboui ; A. Saidani, I. farhat, Z. Bokal, D. Cherif.
}

Department of general surgery-Hopital Mahmoud El Matri -Ariana- Tunisie.

University of Medicine of Tunis El manar - Tunisia.

\section{ABSTRACT}

Duodenal duplication cysts are rare congenital anomalies usually found in children, but rare cases have been described in adults.

We report the case of a duodenal duplication cyst in a 54-yearold man who presented with a gastrointestinal obstruction inherent to endoluminal hemorrhage. Diagnosis was evocated by endoscopy. It was confirmed by computed tomography, oesogasto duodenal swallowing and magnetic resonance imaging.

Partial cystectomy with mucosal stripping was performed via a midline laparotomy. The patient's recovery was uneventful. The diagnosis was confirmed by histological examination of the operative specimen.

In elderly patients with atypical presentation associated to cystic structures neighboring the duodenum, a high index of suspicion is required to diagnose this rare congenital pathology. Complete excision is the optimal treatment, but in some cases, partial cystectomy or endoscopic procedures can be performed.
*Correspondence to Author:

S. Jarboui

Department of general surgery-Hopital Mahoud El-matri-Ariana-Tunisie.

Phone/ 0021698339887

Mail:drslimjarboui @yahoo.fr How to cite this article:

Jarboui et al. Duodenal duplication cyst: a rare cause of gastro-intestinal obstruction. International Journal of Case Reports, 2017 1:6

\section{eSciencePublisher}

eSciPub LLC, Houston, TX USA. Website: http://escipub.com/

Key-words: duplication, duodenal, congenital, excision. 


\section{Introduction:}

Intestinal duplications are mostly diagnosed in children, and less than $30 \%$ of all cases are diagnosed in adults [1,2]. Duodenal duplications (DD) are extremely rare and comprise less than $5 \%$ of all intestinal duplications.

We report a rare case of DD in an adult man revealed by unusual manifestation of GI bleeding with vomiting and duodenal obstruction. Diagnosis was confirmed by imaging and operative findings with subsequent histopathological examination.

\section{Case report:}

A 54-year-old man with no medical history sought medical consultation because of two months history of recurrent vomiting. He was referred to us with the diagnosis of gastric outlet obstruction. Physical examination revealed nothing significant. Laboratory examination revealed functional renal insufficiency without electrolytic perturbations and normocytic anemia, with hemoglobin level of $10.5 \mathrm{gr} / \mathrm{dl}$. Endoscopy after stomach aspiration revealed that the second portion of the duodenum was narrowing because of extralunminal compression by an oval "blue"cyst formation. Computed tomography with contrast injection and ingestion showed an extralunminal cyst formation developing in the lateral wall of the second duodenum wall compressing it. This cyst formation didn't communicate with the digestive luminal and extending until the third portion of the duodenum. The gallbladder and the extra hepatic biliary tract were not distended (Fig1). A complement by MRI had confirmed the cystic nature of the formation depending in the external wall of the second portion of the duodenum, without anatomic relationship with the terminal portion of the extra hepatic biliary tract and the vaterian ampulla (Fig.2). The lesion seemed to be causing narrowing of the duodenal lumen. At laparotomy, after a kocher maneuver, investigation disclosed a cyst structure $4 \mathrm{~cm}$ length located in the external lateral portion of the second duodenum (Fig. 3). After duodenotomy, we confirmed no communication with the bile duct or adjacent pancreas was identified. The lateral part of the cyst was excised and the remnant mucosa directly attached to the duodenal wall was cauterized. After this, we primarily closed the lining defect in the duodenal wall with a $4 / 0$ vicryl one layer simple suture.

The post operative course was uneventful and the patient was discharged at $4^{\text {th }}$ postoperative day. The pathologic examination showed a compatible duodenal wall with partially denuded epithelium consistent with the diagnosis of DD cysts. Fourteen months after surgery, the patient fill well without complaints.

\section{Discussion:}

Intestinal duplications are very rare congenital anomalies. Duodenal duplications (DD) cysts are the rarest ones with less than $5 \%$ of all intestinal duplications [1,2]. The definition includes a smooth-muscle coat, an alimentary epithelial lining and an intimate attachment to the GI tract. They can be cystic or tubular, communicating or not communicating $[1,3,4]$. Our case report has some particularities owing to its first manifestation in the adulthood, the uncommon clinical presentation with duodenal obstruction caused by endoluminal hemorrhage and the anatomical location in the antimesenteric side of the second duodenum which is very rare.

As we have said, DD cysts are mostly diagnosed in childhood and such a lesion are rarely detected in an adult like in the current case $[1,2,4]$. They have diverse clinical manifestations depending in the location, the presence or not of complications and the relationship with adjacent organ especially the pancreas and the biliary tree. Recurrent nausea, vomiting, abdominal mass, pancreatitis, jaundice can be seen [5-10]. In the systematic review of Chen et al. compiling 47 cases of DD cysts, $80 \%$ of patients presented with abdominal pain and only 18 patients were older than 20 years [1]. In some cases, ectopic gastric mucosa can appear and cause ulceration and lead to bleeding like in our case reported.

Preoperative diagnosis is not easy and is a challenging because the symptoms are no specifics and the entity is very rare. The doubled- layered wall of the duodenum is used to reach a diagnosis. On ultrasonography, we found a mass with an inner hyperchoic rim corresponding to the mucosa and submucosa surrounded by a hypoechoic layer corresponding to the muscularis propria "the Gut Signature". Barium studies of GI demonstrate the mass effect and displacement 


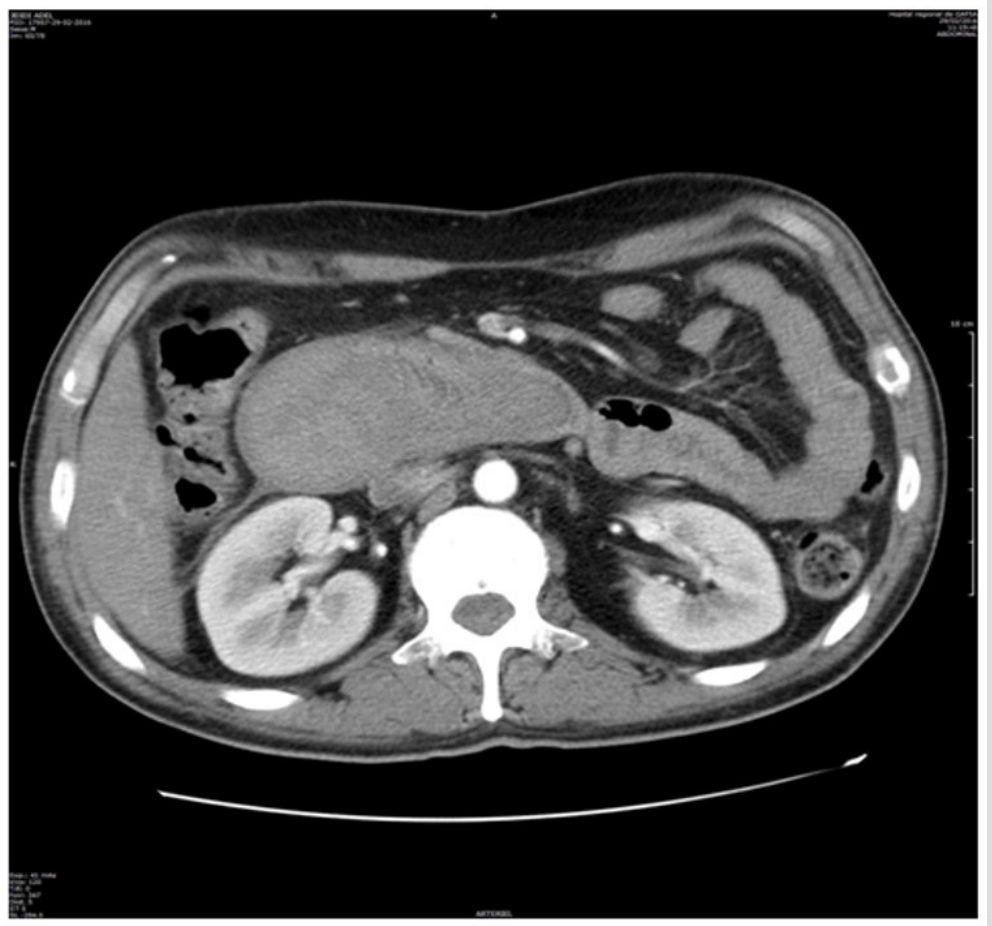

Fig.1: Axial reconstruction computed Tomography with arterial phase: Cyst formation developing in the external side of DIl with spontaneous hyper density due to $\mathrm{Gl}$ bleeding.
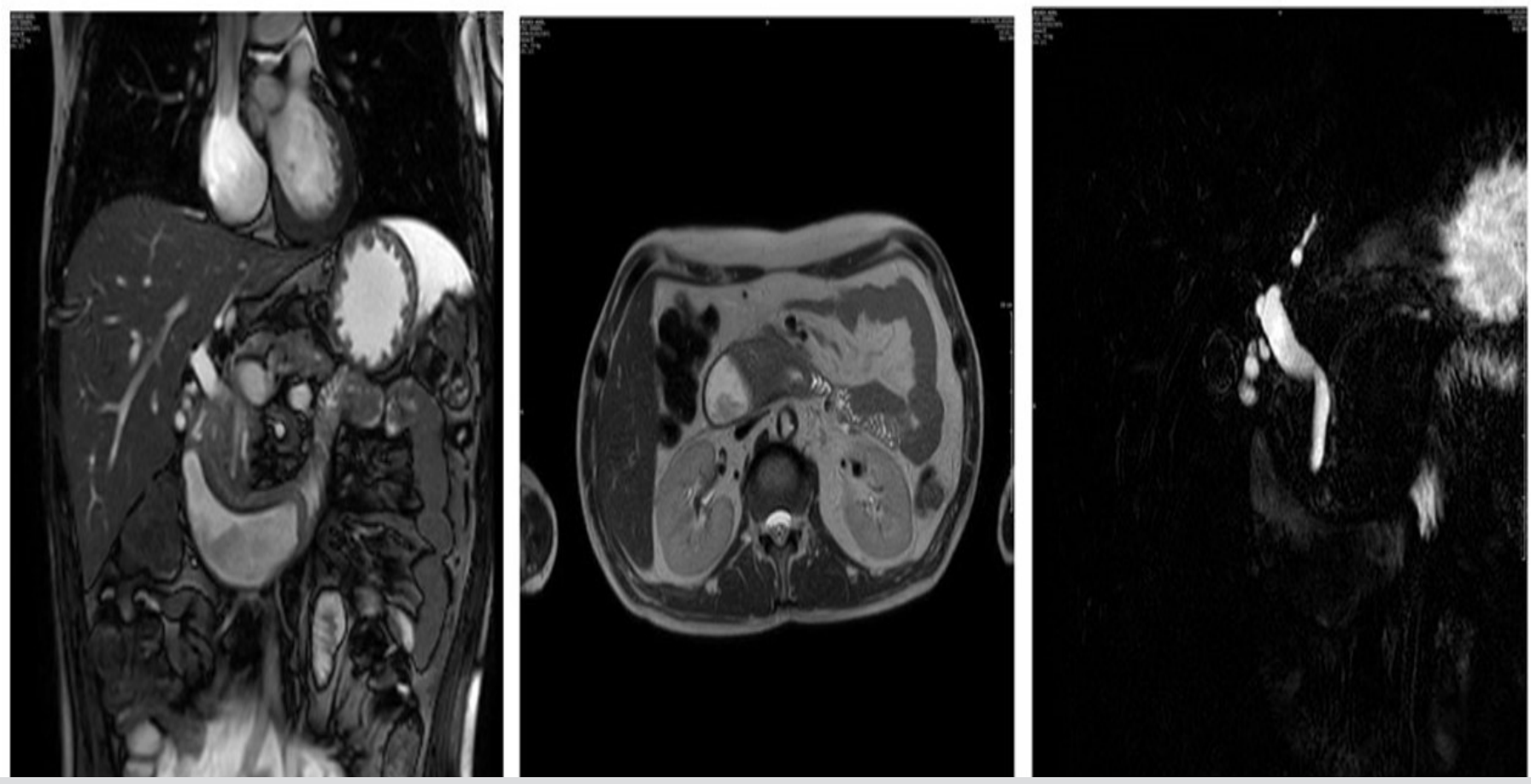

Fig.2: MRI: A- Coronal reconstruction with T2 weighted image demonstrating the external cyst of DIl with heterogeneous content. B: Axial reconstruction T2 weighted image with spontaneous hyper intense fluid content of the duodenal cyst.DII and DIII were internally displaced and compressed by the mass. C: Cholangiopancreatico MRI showing no relationship of the cyst with the Vaterian ampulla or the biliary duct. 
of normal alignment in the non-communicating cyst, but in the communicating type, the cyst itself is being filled with barium $[1,4,7,9]$. In our case, the diagnosis was firstly suspected by the endoscopy that showed a blue- external cyst in the external part of the DII narrowing the lumen and causing duodenal obstruction. The blue aspect is caused by the endoluminal hemorrhage in the cyst. The abdominal computed tomography (CT) with multiplanar reconstruction and Magnetic Resonance Imaging (MRI) have facilitated the diagnosis and the evaluation of the relationship with the pancreas and the biliary tree so, we can speculated the best management (surgical or endoscopic approach). Some authors advocated the need for preoperative or per operative cholangiopancreatography to exclude bilio-pancreatic communication with the duplication cyst []. In our case, despite the unusual location of the DD cysts in the lateral part of DII, MRI didn't found any communication or relationship with the papilla of Vater or bile duct. Differential diagnosis should be mindful of choledocele, pancreatic pseudocyst, cystic dystrophy of duodenal wall or intraluminal diverticulum $[1,10,11]$.

Management of the DD cysts depends on the location and proximity to the duodenal wall, the pancreas or biliary tracts. Surgery for symptomatic or complicated lesions is always justified. For those asymptomatic the indications remain controversial, but for us they are justified because the risk of malignancy and complications particularly in the gastric ectopic mucosa (ulceration, bleeding, perforation) $[1,7,11]$. The ideal treatment of DD cysts is complete surgical resection if their locations allow it without damaging the neighboring structures. For the cysts that involve the pancreatic head and biliary tract, the treatment of choice is pancreaticoduodenectomy. In our case, because its location in the external side of the duodenal wall, partial cystectomy with mucosa cauterization was performed.

Some case or laparoscopic approach was reported particularly for DD Cysts in their extra luminal locations [12]. For those locations, endoscopic internal derivation or marsupialization cannot be performed because cannot remove the mucosal layer. But, for communicating and intraluminal types, endoscopic internal derivation was found to be effective and avoid the morbidity associated with surgery [4]. However, the natural history of DD cysts remain uncertain and some case of malignancy have been reported so, endoscopy and biopsy is recommended after treatment and in the presence of ectopic gastric mucosa, surgery is recommended because of the risk of subsequent hemorrhage $[11,13]$.

\section{Conclusion:}

Duodenal duplication is very rare congenital malformation. Diagnosis should be considered even in elderly patients who present with abdominal symptoms and cystic lesion neighboring the duodenum on radiology. Total excision is the choice of treatment, but partial cystectomy or internal derivation by endoscopy should be considered in certain scenarios.

\section{References:}

1- Chen J, Lee H, Yeung C, Chan W, Jiang C, Sheu J. Meta-analysis: the clinical features of the duodenal duplication cyst. J of Pediatric Surg 2010; 45: 1598-1606.

2- Chang H, Huang S, Wu R, Yu M. Duodenal duplication cyst: A rare cause of geriatric gastrointestinal bleeding. $\mathrm{J}$ of the Chineese Medical Association 2001; 74: 233-236.

3- Uzun M.A, Koksal N, Kayahan M, Celik A, Kiliciglu G, Ozkara S. A rare case of duodenal duplication treated surgically. World J of Gastroenterol 2009; 15: 882-884.

4- J Rocks M.A, McAlister V.C. Endoscopic Fenestration of a duodenal Duplication cyst to resolve Recurrent Pancreatitis. JOP 2007; 8: 795798.

5- Thorpe M.A, Nijagal A, Mooney D. Surgical management of an intussuscepted duodenal duplication cyst in a pediatric patient with heterotaxy. J Ped Surg Case Reports 2015; 3: 455-458.

6- Jadlowiec C.C, Lobel B.E, Akolkar N, Bourque M.D, Devers T.J, McFadden D.W. Presentation and surgical Management of Duodenal Duplication in Adults. Case Rep Surg 2015:659150. doi: $10.1155 / 2015 / 659150$.

7- Seeliger B, Piardi T, Marzano E, Mutter D, Marescaux J, Pessaux P. Duodenal Duplication Cyst: A Potentially Malignant Disease. Ann Surg oncol 2012: 19 : 3753-3754.

8- Salemis N.S, Liatsos C, Kolios M, Gourgiotis S. Recurrent acute pancreatitis secondary to a duodenal duplication cyst in an adult. A case report and literature review. Can J Gastroenterol 
2009; 23: 750-752.

9- Antaki N, Abboud D, Lemmers A, A ntaki F, Deviere J. Acute recurrent pancreatitis secondary to the rare association of a duodenal duplication cyst and a pancreas divisum. Clin Research Hepatol Gastroenterol 2013; 37: 32-36.

10- Yan X, Fan Y, Wang K, Zhang W, Song Y. Duodenal duplication manifested by abdominal pain and bowel obstruction in an adolescent: a case report. Int J Clin Exp med 2015: 8: 2171021714.

11- Bong J.J, Spalding D. Duodenal Duplication Cyst (DDC) communicating with the Pancreatobiliary Duct: A rare Cause of Recurrent Acute Pancreatitis in Adults. J Gastrintest Surg 2010; 14: 199-202.

12- Byun J, Oh H, Kim S, Jung H, JUNG S, Park K, Kim W. Laparoscopic partial cystectomy with mucosal stripping of extraluminal duodenal duplication cysts. World J of Gastroenterol 2014; 20: 1123-

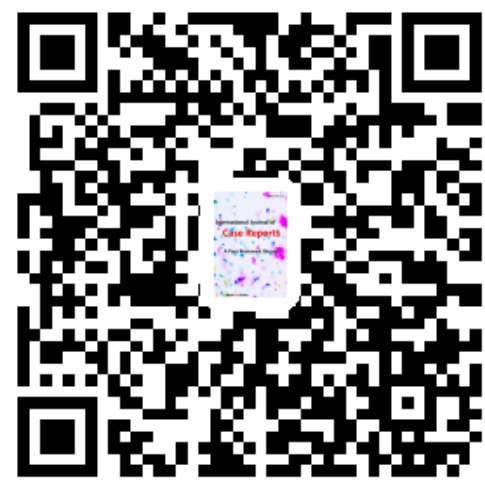
1126.

13- Yamauchi Y, Hoshimo S, Yamashita Y, Funamoto S, Ishida K, Shirakusa T. Successful resection of an infected Duodenal Duplication Cyst after percutaneous Cyst Drainage: Report of a case. Surg Today 2005; 35: 586-589.
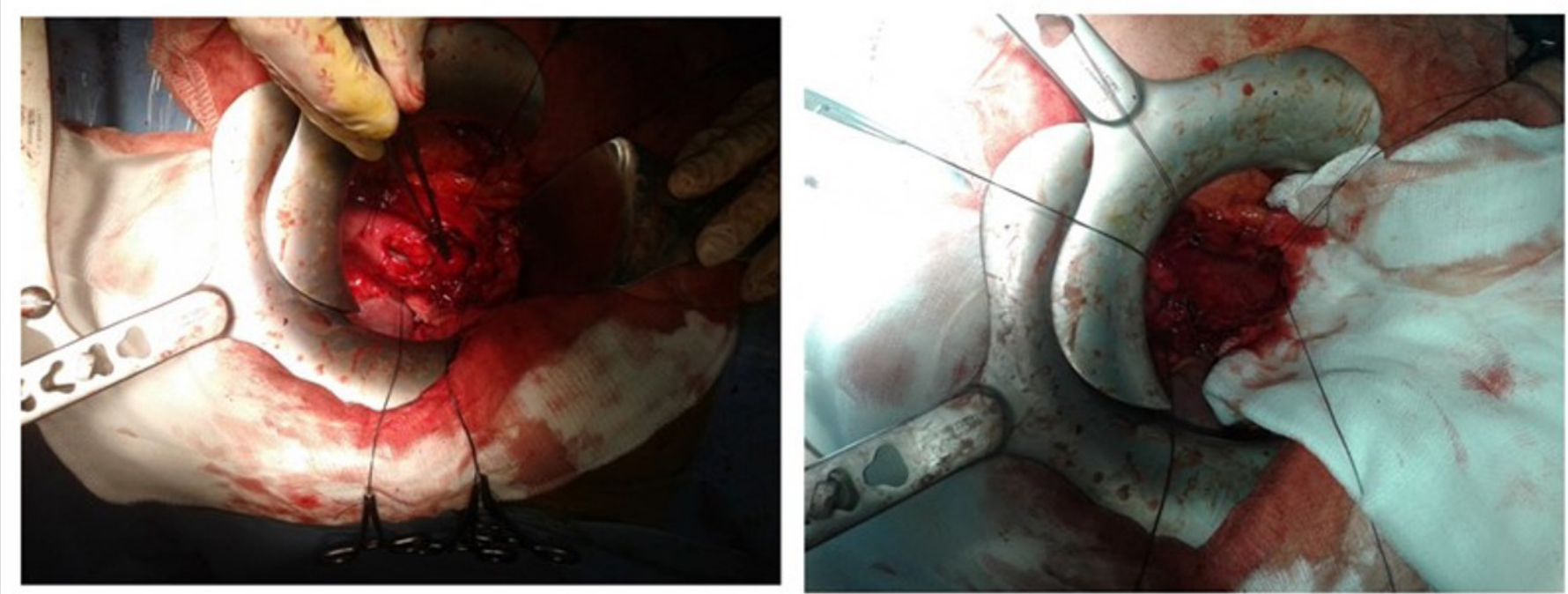

Fig.3: Operative view with the stitches exposing the edges of the duodenal duplication. 